\title{
Unicity in Approximation of a Function and its Derivatives
}

\section{By Lee Johnson}

For $f$ continuous and real on $[0,1]$, let $\|f\|=\max |f(x)|, x \in[0,1]$. In this journal, Moursund [3] proved

Theorem 1. Let $f$ be twice differentiable on $[0,1]$. Among all polynomials $h(x)$ of degree $n$ or less, let $p(x)$ be the one that minimizes: $\max \left\{\|h-f\|,\left\|h^{\prime}-f^{\prime}\right\|\right\}$. If $q(x)$ is another such minimizing polynomial, then $q^{\prime}=p^{\prime}$.

Let $f^{i}$ denote the $i$ th derivative of $f$. Moursund's result can be extended to:

Theorem 2. Let $f$ be $(k+1)$-times differentiable on $[0,1]$. Among all polynomials $h(x)$ of degree $n$ or less, let $p(x)$ be the one that minimizes:

$$
\max \left\{\|h-f\|,\left\|h^{1}-f^{1}\right\|, \cdots,\left\|h^{k}-f^{k}\right\|\right\} .
$$

If $q(x)$ is another such minimizing polynomial, then $q^{k}=p^{k}$.

We need some preliminary results before establishing Theorem 2. Let $M(h)=$ $\max \left\{\|h\|, \cdots,\left\|h^{k}\right\|\right\}$. The functional $M$ is a norm on the set $S$ of functions that are $(k+1)$-times differentiable on $[0,1]$.

Let $Q$ denote the set of polynomials of degree $n$ or less. Call $p_{0} \in Q$ a best approximation to $f \in S$ if $M\left(p_{0}-f\right) \leqq M(q-f)$, for all $q \in Q$. It can be shown [1] that the set of best approximations is convex and nonempty.

Call $x \in[0,1]$ an extreme point of $p-f$ if for some $i, \quad 0 \leqq i \leqq k$, $\left|p^{i}(x)-f^{i}(x)\right|=|| p^{i}-f^{i} \|=M(p-f)$. Denote the set of extreme points of $p-f$ by $E(p, f)$. Standard arguments quickly show [2] that $p$ is a best approximation to $f$ if and only if $p$ is a best approximation to $f$ on $E(p, f)$.

Proof of Theorem 2. Let $p$ and $q$ be two best approximations to $f$; and suppose $p^{k} \neq q^{k}$. Let $c=t p+(1-t) q, t \in(0,1)$; then $c$ is also a best approximation to $f$. Using $p^{k} \neq q^{k}$, we will construct an approximation to $f$ that is better than $c$ on $E(c, f)$, giving a contradiction. Let $a_{i}=j$ if there are $j$ points $x$ in $(0,1)$ such that $\left|c^{i}(x)-f^{i}(x)\right|=\left\|c^{i}-f^{i}\right\|=M(c-f)$.

Let $b_{i}=0,1,2$ according as none, one or both of $z=0, z=1$ are such that $\left|c^{i}(z)-f^{i}(z)\right|=M(c-f)$. In particular, $a_{i}=b_{i}=0$ if $\left\|c^{i}-f^{i}\right\|<M(c-f)$.

If $x_{0}$ is among the $a_{i}$ extreme points of $c^{i}-f^{i}$, then

(1) $x_{0}$ is not among the $a_{i+1}$ extreme points of $c^{i+1}-f^{i+1}$,

(2) $p^{i}\left(x_{0}\right)-f^{i}\left(x_{0}\right)=q^{i}\left(x_{0}\right)-f^{i}\left(x_{0}\right)= \pm M(c-f)$,

(3) $p^{i+1}\left(x_{0}\right)-f^{i+1}\left(x_{0}\right)=q^{i+1}\left(x_{0}\right)-f^{i+1}\left(x_{0}\right)=0$.

From (2) and (3), $p^{i}(x)-q^{i}(x)$ has at least $2 a_{i}+b_{i}$ zeroes. We will show that $p^{i}-q^{i}$ has at least $\left(b_{0}+\cdots+b_{i}\right)+2\left(a_{0}+\cdots+a_{i}\right)-i$ zeroes.

Lемма. Let $h(x)$ be a polynomial with $r$ single zeroes, $s$ double zeroes and $t$ triple zeroes. Let $h^{\prime}(x)$ have $u$ double zeroes-none of which are among the triple zeroes of $h(x)$. Then $h^{\prime}(x)$ has at least $r+2 s+3 t+2 u-1$ zeroes.

Proof. Let $r+s+t=v$, and label the zeroes of $h(x)$ as $x_{1}, \cdots, x_{v} ; x_{i}<x_{i+1}$.

Received November 16, 1967. 
In $\left(x_{i}, x_{i+1}\right)$ there is a zero of $h^{\prime}(x)$; furthermore, this zero must be of odd multiplicity. Also none of the $u$ double zeroes of $h^{\prime}(x)$ are counted among the $v$ distinct zeroes of $h(x)$. Counting the zeroes of $h^{\prime}(x)$ we obtain

(a) $s+2 t$; from the multiple zeroes of $h(x)$,

(b) $v-1$; the zeroes of $h^{\prime}(x)$ in $\left(x_{i}, x_{i+1}\right)$,

(c) $2 u$; as noted, the $v-1$ zeroes in (b) are of odd multiplicity. If one of the $u$ double zeroes of $h^{\prime}(x)$ is included in (b), this zero must have been of multiplicity 3 or more.

Adding (a), (b) and (c), establishes the lemma.

Using (1), (2) and (3) from above; and applying the lemma repeatedly to the derivatives of $p(x)-q(x)$, we obtain

(4) $p^{i}(x)-q^{i}(x)$ has at least $\left(b_{0}+\cdots+b_{i}\right)+2\left(a_{0}+\cdots+a_{i}\right)-i$ zeroes.

As $p^{k}-q^{k} \neq 0$, it must be that $n-k \geqq\left(b_{0}+\cdots+b_{k}\right)+2\left(a_{0}+\cdots+a_{k}\right)$ $-k$.

The same argument, starting with $p^{j}(x)-q^{j}(x)$, gives that $p^{k}-q^{k}$ has $\left(b_{j}+\cdots+b_{k}\right)+2\left(a_{j}+\cdots+a_{k}\right)-(k-j)$ zeroes. Thus, $p^{k} \neq q^{k}$ means that

(5) $\left(b_{j}+\cdots+b_{k}\right)+2\left(a_{j}+\cdots+a_{k}\right) \leqq n-j, 0 \leqq j \leqq k$.

We will use (5) to construct a polynomial $r(x)$ such that $r^{i}(y)-f^{i}(y)=0$ if $y$ is one of the $a_{i}+b_{i}$ extreme points of $c^{i}-f^{i}$. Select $s$ points in $(0,1)$, distinct from the $a_{0}+b_{0}$ extreme points of $c-f$; where $s+\left(b_{0}+\cdots+b_{k}\right)+$ $2\left(a_{0}+\cdots+a_{k}\right)=n+1$. Note that from $(5), s \geqq 1$.

Let $D(x)=\left(1, x, x^{2}, \cdots, x^{n}\right), D^{1}(x)=\left(0,1,2 x, \cdots, n x^{n-1}\right), D^{2}(x)=$ $\left(0,0,2, \cdots, n(n-1) x^{n-2}\right)$. Define $D^{i}$ similarly, $i=3, \cdots, k$. We will form an $(n+1) \times(n+1)$ "Vandermonde-like" matrix $A$, as follows. For each of the $s$ points $y_{1}, \cdots, y_{s}$ chosen in $(0,1)$, let $A$ have a row of the form $D\left(y_{i}\right)$. For each of the $a_{0}$ extreme points $w$ of $c-f$, let $A$ have two rows of the form

$$
\begin{aligned}
& D(w) \\
& D^{\prime}(w)
\end{aligned}
$$

lor each of the $b_{0}$ "end-point" extreme points $z$ of $c-f$, let $A$ have a row of the form $D(z)$.

Generally, for each of the $a_{i}$ extreme points $w$ of $c^{i}-f^{i}$, let $A$ have 2 rows of the form

$$
\begin{aligned}
& D^{i}(w) \\
& D^{i+1}(w)
\end{aligned}
$$

For each of the $b_{i}$ "end-point" extreme points of $c^{i}-f^{i}$, let $A$ have a row of the form $D^{i}(z)$.

We now show that $A$ is nonsingular. Suppose $A d^{T}$ is the zero vector; where $d=\left(d_{0}, d_{1}, \cdots, d_{n}\right)$. Form $h(x)=d_{n} x^{n}+\cdots+d_{1} x+d_{0}$. Clearly, $h^{i}(x)$ has $a_{i}$ double zeroes and $b_{i}$ single zeroes; $0 \leqq i \leqq k$. Applying the lemma, $h^{k}(x)$ has $s+\left(b_{0}+\cdots+b_{k}\right)+2\left(a_{0}+\cdots+a_{k}\right)-k=n+1-k$ zeroes. As $h^{k}(x)$ has degree $n-k$ or less, $h^{k}=0$.

Using (5), and $s+\left(b_{0}+\cdots+b_{k}\right)+2\left(a_{0}+\cdots+a_{k}\right)=n+1$, we have

$$
s+\left(b_{0}+\cdots+b_{j}\right)+2\left(a_{0}+\cdots+a_{j}\right) \geqq j+2 ; j=0,1, \cdots, k-1 .
$$

Hence by (4), $h^{j}(x)$ has at least 2 zeroes, $j=0,1, \cdots, k-1$. As $h^{k}=0$, this 
shows that $h=0$, or $d_{i}=0, i=0,1, \cdots, k$. Thus, $A$ is nonsingular.

As $A$ is nonsingular, we can fit $f(x), f^{1}(x), \cdots, f^{k}(x)$ exactly on the $\left(b_{0}+\cdots+b_{k}\right)$ $+2\left(a_{0}+\cdots+a_{k}\right) \leqq n$ extreme points of $c-f$. That is, we can find $r(x)$ of degree $n$ or less, so that if $\left|c^{i}\left(x^{\prime}\right)-f^{i}\left(x^{\prime}\right)\right|=\left\|c^{i}-f^{i}\right\|=M(c-f)$, then $r^{i}\left(x^{\prime}\right)-f^{i}\left(x^{\prime}\right)=0$. It may well be, even though $r^{i}\left(x^{\prime}\right)-f^{i}\left(x^{\prime}\right)=0$, that $\left|r^{j}\left(x^{\prime}\right)-f^{j}\left(x^{\prime}\right)\right| \geqq M(c-f)$ for some $j, j \neq i$. If this is the case, $x^{\prime}$ must not have been one of the $a_{j}+b_{j}$ extreme points of $c^{j}-f^{i}$. If $\left|c^{j}\left(x^{\prime}\right)-f^{i}\left(x^{\prime}\right)\right|<M(c-f)$, there is some $t \in(0,1)$ such that

$$
\left|t\left(r^{i}\left(x^{\prime}\right)-f^{i}\left(x^{\prime}\right)\right)+(1-t)\left(c^{i}\left(x^{\prime}\right)-f^{i}\left(x^{\prime}\right)\right)\right|<M(c-f) .
$$

As $E(c, f)$ was supposed to be a finite set, we can use the above remark to choose some $t \in(0,1)$ such that

$$
\left|t\left(r^{i}(x)-f^{i}(x)\right)+(1-t)\left(c^{i}(x)-f^{i}(x)\right)\right|<M(c-f), \text { for all } x \in E(c, f),
$$

$i=0,1, \cdots, k$. This gives $t r+(1-t) c$ a better approximation to $f$ on $E(c, f)$ than is $c$. Thus, $c$ could not have been a best approximation.

The proof above, except for cumbersome notational modifications, clearly establishes the more general

Тнеовем 3. Let $i, j, \cdots, k$ be any finite sequence of nonnegative integers, $i<j<\cdots<k$. Let $f(x)$ be $(k+1)$-times differentiable on $[a, b]$. Among all polynomials $h(x)$ of degree $n$ or less, let $p(x)$ be one that minimizes:

$$
\max \left\{\left\|h^{i}-f^{i}\right\|,\left\|h^{i}-f^{i}\right\|, \cdots,\left\|h^{k}-f^{k}\right\|\right\} .
$$

If $q(x)$ is another such minimizing polynomial, then $q^{k}=p^{k}$.

Virginia Polytechnic Institute

Department of Mathematics

Blacksburg, Virginia 24061

1. R. E. Langer (Editor), On Numerical Approximation, Univ. of Wisconsin Press, Madison, Wis., 1959, pp. 11-23.

2. L. W. Johnson, Approximation of Vector-Valued Functions, Dissertation, Michigan State Univ., East Lansing, Mich., 1967.

3. I. G. Moursund, "Chebyshev approximation of a function and its derivatives," Math. Comp., v. 18, 1964, pp. 382-389. 